Pathologe $2017 \cdot 38: 545-546$

https://doi.org/10.1007/s00292-017-0372-z

Online publiziert: 16. Oktober 2017

๑) Springer Medizin Verlag GmbH 2017

CrossMark
A. Staebler ${ }^{1} \cdot$ D. Mayr ${ }^{2}$

${ }^{1}$ Institut für Pathologie und Neuropathologie, Universitätsklinikum Tübingen, Eberhard-Karls-Universität, Tübingen, Deutschland

${ }^{2}$ Pathologisches Institut, Ludwig-Maximilians-Universität München, München, Deutschland

\section{Erratum zu: Update der S3-Leit- linie für maligne Ovarialtumoren 2016}

\section{Rolle der Pathologie in Diagnostik, Therapie und Nachsorge epithelialer Tumoren}

\section{Erratum zu:}

\section{Pathologe 2017}

https://doi.org/10.1007/s00292-017-

0310-0

Im CME-Beitrag,„Update der S3-Leitlinie für maligne Ovarialtumoren 2016. Rolle der Pathologie in Diagnostik, Therapie und Nachsorge epithelialer Tumoren“, wurden die TNM-Klassifikation (2017) und FIGO-Klassifikation in - Tab. 5 leider unvollständig abgedruckt. Des Weiteren fehlt im Textabschnitt „Muzinöses Karzinom“ die Angabe, dass eine starke CDX2- und CK20-Positivität bei CK7-Negativität oder schwacher Positivität sowie ein knotiges Wuchsmuster für eine metastatische Infiltration durch ein Karzinom des Kolons sprechen. Wir bitten, die korrekten Angaben zur Histomorphologie von primär muzinösen Ovarialkarzinomen sowie die vollständige $\bullet$ Tab. $5 \mathrm{zu}$ beachten.

\section{Korrespondenzadresse}

\section{PD Dr. A. Staebler}

Institut für Pathologie und Neuropathologie, Universitätsklinikum Tübingen, Eberhard-KarlsUniversität

Liebermeisterstraße 8, 72076 Tübingen,

Deutschland

annette.staebler@med.uni-tuebingen.de 


\section{Erratum}

Tab. 5 TNM-Klassifikation (2017) und FIGO-Klassifikation

\begin{tabular}{|c|c|c|}
\hline TNM & FIGO & Definition \\
\hline TX & \multicolumn{2}{|c|}{ Primärtumor nicht bekannt, keine Angaben möglich } \\
\hline T0 & \multicolumn{2}{|c|}{ Kein Anhalt für einen Tumor } \\
\hline T1 & 1 & Tumor auf die Ovarien oder Tuben beschränkt \\
\hline T1a & IA & $\begin{array}{l}\text { Auf ein Ovar (Kapsel intakt) oder eine Tube (Serosa intakt) be- } \\
\text { schränkt, Ovar- oder Tubenoberfläche tumorfrei, negative Spülzy- } \\
\text { tologie }\end{array}$ \\
\hline $\mathrm{T} 1 \mathrm{~b}$ & IB & $\begin{array}{l}\text { Befall beider Ovarien (Kapsel intakt) oder beider Tuben (Serosa in- } \\
\text { takt), Ovar- oder Tubenoberfläche tumorfrei, negative Spülzytologie }\end{array}$ \\
\hline T1c & IC & $\begin{array}{l}\text { Tumor befällt ein oder beide Ovarien oder Tuben mit Nachweis einer } \\
\text { der folgenden Punkte: }\end{array}$ \\
\hline T1c1 & IC1 & latrogene Kapsel-(Serosa-)Ruptur \\
\hline T1c2 & IC2 & $\begin{array}{l}\text { Präoperative Kapsel-(Serosa-)Ruptur oder Tumor auf der Ovar- oder } \\
\text { Tubenoberfläche }\end{array}$ \\
\hline T1c3 & IC3 & Maligne Zellen im Aszites oder in der Spülzytologie nachweisbar \\
\hline $\mathrm{T} 2$ & II & $\begin{array}{l}\text { Tumor befällt ein oder beide Ovarien oder Tuben mit zytologisch } \\
\text { oder histologisch nachgewiesener Ausbreitung in das kleine Becken } \\
\text { oder primäres Peritonealkarzinom }\end{array}$ \\
\hline $\mathrm{T} 2 \mathrm{a}$ & $\| A$ & $\begin{array}{l}\text { Ausbreitung und/oder Tumorimplantate auf Uterus und/oder Tuben } \\
\text { und/oder Ovarien }\end{array}$ \\
\hline $\mathrm{T} 2 \mathrm{~b}$ & IIB & $\begin{array}{l}\text { Ausbreitung auf weitere intraperitoneale Strukturen im Bereich des } \\
\text { kleinen Beckens }\end{array}$ \\
\hline $\begin{array}{l}\text { T3 und/oder } \\
\text { N1 }\end{array}$ & III & $\begin{array}{l}\text { Tumor befällt ein oder beide Ovarien oder Tuben oder primäres Pe- } \\
\text { ritonealkarzinom mit zytologisch oder histologisch nachgewiesener } \\
\text { Ausbreitung außerhalb des kleinen Beckens und/oder regionäre } \\
\text { LK-Metastasen }\end{array}$ \\
\hline N1 & IIIA1 & Nur retroperitoneale LK-Metastasen \\
\hline N1a & IIIA1i & LK-Metastasen $\leq 10 \mathrm{~mm}$ \\
\hline N1b & IIIAii & LK-Metastasen $>10 \mathrm{~mm}$ \\
\hline T3a Jedes $N$ & IIIA2 & $\begin{array}{l}\text { Mikroskopische Ausbreitung jenseits des Beckens mit oder ohne } \\
\text { retroperitoneale LK-Metastasen jenseits des Beckens }\end{array}$ \\
\hline T3b Jedes $N$ & IIIB & Makroskopische Ausbreitung jenseits des Beckens $\leq 2 \mathrm{~cm}$ \\
\hline T3c Jedes $N$ & IIIC & $\begin{array}{l}\text { Makroskopische Ausbreitung jenseits des Beckens }>2 \mathrm{~cm} \text { und/oder } \\
\text { regionäre LK-Metastasen; einschließlich Tumorausbreitung auf die } \\
\text { Leber- und/oder Milzkapsel ohne Parenchymbeteiligung }\end{array}$ \\
\hline M1 & IV & Fernmetastasen mit Ausnahme peritonealer Metastasen \\
\hline M1a & IVA & Pleuraerguss mit positiver Zytologie \\
\hline M1b & IVB & $\begin{array}{l}\text { Parenchymale Metastasen der Leber und/oder der Milz, Metastasen } \\
\text { in extraabdominellen Organen (einschließlich inguinaler LK und LK } \\
\text { außerhalb des Abdomens) }\end{array}$ \\
\hline $\begin{array}{l}\text { FIGO "Inte } \\
\text { se, } L K \text { Lym }\end{array}$ & a $\mathrm{Fe}$ & n of Gynecology and Obstetrics", TNM Tumor, Lymphknoten, Metasta- \\
\hline
\end{tabular}

